\title{
Probing neutrino nonstandard interactions with atmospheric neutrino data
}

\author{
N. Fornengo \\ Dipartimento di Fisica Teorica, Università di Torino and INFN, Sezione di Torino, Via Pietro Giuria 1, I-10125 Torino, Italy \\ M. Maltoni, R. Tomàs Bayo, and J. W. F. Valle \\ Instituto de Física Corpuscular-CSIC/Universitat de València, Edificio Institutos de Paterna, Apt 22085, E-46071 Valencia, Spain
}

(Received 9 August 2001; published 12 December 2001)

\begin{abstract}
We reconsider the atmospheric neutrino anomaly in light of the latest data from Super-Kamiokande contained events and from Super-Kamiokande and MACRO upgoing muons. We reanalyze the proposed solution to the atmospheric neutrino anomaly in terms of nonstandard neutrino-matter interactions (NSI's) as well as the standard $\nu_{\mu} \rightarrow \nu_{\tau}$ oscillations (OSC's). Our statistical analysis shows that a pure NSI mechanism is now ruled out at $99 \%$, while the standard $\nu_{\mu} \rightarrow \nu_{\tau}$ OSC mechanism provides a quite remarkably good description of the anomaly. We therefore study an extended mechanism of neutrino propagation which combines both oscillation and nonstandard neutrino-matter interactions, in order to derive limits on flavor-changing and nonuniversal neutrino interactions. We obtain that the off-diagonal flavor-changing neutrino parameter $\varepsilon$ and the diagonal nonuniversality neutrino parameter $\varepsilon^{\prime}$ are confined to $-0.05<\varepsilon<0.04$ and $\left|\varepsilon^{\prime}\right|<0.17$ at $99 \%$ C.L. These limits are model independent and they are obtained from pure neutrino-physics processes. The stability of the neutrino oscillation solution to the atmospheric neutrino anomaly against the presence of nonstandard neutrino interactions establishes the robustness of the near-maximal atmospheric mixing and massive-neutrino hypothesis. The best agreement with the data is obtained for $\Delta m^{2}=2.4 \times 10^{-3} \mathrm{eV}^{2}, \sin ^{2}(2 \theta)=0.99, \varepsilon=-9.1$ $\times 10^{-3}$, and $\varepsilon^{\prime}=-1.9 \times 10^{-3}$, although the $\chi^{2}$ function is quite flat in the $\varepsilon$ and $\varepsilon^{\prime}$ directions for $\varepsilon, \varepsilon^{\prime} \rightarrow 0$.

DOI: 10.1103/PhysRevD.65.013010

PACS number(s): 14.60.St, 13.15.+g, 14.60.Pq
\end{abstract}

\section{INTRODUCTION}

The experimental data on atmospheric neutrinos [1-4] show, in the muon-type events, a clear deficit which cannot be accounted for without invoking nonstandard neutrino physics. This result, together with the solar neutrino anomaly [5], is very important since it constitutes clear evidence for physics beyond the standard model. Altogether, the simplest joint explanation for both solar and atmospheric anomalies is the hypothesis of three-neutrino oscillations [6].

There are however many attempts to account for neutrino anomalies without oscillations [7]. Indeed, in addition to the simplest oscillation interpretation [8,9], the solar neutrino problem offers very good alternative explanations, for example based on transition magnetic moments [10] or nonstandard neutrino interactions (NSI's) [11]. Likewise, several such alternative mechanisms have been postulated to account for the atmospheric neutrino data such as the NSI's [12] or the neutrino decay hypotheses [13]. ${ }^{1}$

In contrast with the solar case, the atmospheric neutrino anomaly is so well reproduced by the $\nu_{\mu} \rightarrow \nu_{\tau}$ oscillation (OSC) hypothesis $[17,19]$ that one can use the robustness of this interpretation so as to place stringent limits on a number of alternative mechanisms. Among the various proposed alternative interpretations, one possibility is that the neutrinos possess nonstandard interactions with matter, which were shown to provide a good description of the contained event data sample [12]. Such nonstandard interactions [20-22] can be either flavor-changing (FC) or nonuniversal (NU), and

\footnotetext{
${ }^{1}$ For more exotic attempts to explain the neutrino anomalies see Refs. [14-16].
}

arise naturally in theoretical models for massive neutrinos [23-29]. This mechanism does not even require a mass for neutrinos $[24,25]$ although neutrino masses are expected to be present in most models [23,26-30]. It is therefore interesting to check whether the atmospheric neutrino anomaly could be ascribed, completely or partially, to nonstandard neutrino-matter interactions. In Refs. $[12,31,32]$ the atmospheric neutrino data have been analyzed in terms of a pure $\nu_{\mu} \rightarrow \nu_{\tau}$ conversion in matter due to NSI's. The disappearance of $\nu_{\mu}$ from the atmospheric neutrino flux is due to interactions with matter which change the flavor of neutrinos. A complete analysis of the 52 kton yr Super-Kamiokande data was given in Ref. [31]. It included both the low-energy contained events as well as the higher-energy stopping and through-going muon events, and showed that the NSI solution was acceptable, although the statistical relevance was low. Compatibility between the data and the NSI hypothesis was found to be $9.5 \%$ for relatively large values of flavorchanging and nonuniversality parameters. ${ }^{2}$

In the present paper we will use the latest higher statistics data from Super-Kamiokande (79 kton yr) [3] and MACRO [33] data in order to briefly reanalyze the atmospheric data within the oscillation hypothesis. We show that the oscillation description has a high significance, at the level of $99 \%$ for the Super-Kamiokande data, and 95\% when the MACRO through-going muons data are also added to the analysis. We then show that the new data rule out the NSI mechanism as the dominant conversion mechanism. The goodness of the fit (GOF) is now lowered to $1 \%$. This clearly indicates that a

\footnotetext{
${ }^{2}$ For another analysis showing low confidence for a dominant NSI in atmospheric neutrinos, see Ref. [18].
} 
pure NSI mechanism cannot account for the atmospheric neutrino anomaly.

However, the possibility that neutrinos both possess a mass and nonstandard interactions is an intriguing possibility. For example in models where neutrinos acquire a mass in seesaw type schemes the neutrino masses naturally come together with some nondiagonality of the neutrino states [23]. Alternatively, in supersymmetric models with breaking of $R$ parity [27] neutrino masses and flavor-changing interactions coexist. ${ }^{3}$ This in turn can induce some amount of flavorchanging interactions. The combined mechanism of OSC's together with NSI's may be active in depleting the atmospheric $\nu_{\mu}$ flux, and therefore it can provide an alternative explanation of the deficit. Since the atmospheric neutrino anomaly is explained remarkably well by $\nu_{\mu} \rightarrow \nu_{\tau}$ oscillations, while pure NSI's cannot account for the anomaly, this already indicates that NSI's can be present only as a subdominant channel. The atmospheric neutrino data can therefore be used as a tool to set limits to the amount of NSI's for neutrinos. These limits are obtained from pure neutrinophysics processes and are model independent, since they do not rely on any specific assumption of neutrino interactions. In particular they do not rely on any $S U(2)_{L}$ assumption relating the flavor-changing neutrino scattering off quarks (or electrons) to interactions which might induce anomalous tau decays [35] or suffer from QCD uncertainties. In the following we will show that, from the analysis of the full set of the latest 79 kton yr Super-Kamiokande [3] and the MACRO data on upgoing muons [33] atmospheric neutrino data, FC and nonuniversal neutrino interactions are constrained to be smaller than $5 \%$ and $17 \%$ of the standard weak neutrino interaction, respectively, without any extra assumption.

The plan of the paper is the following. In Sec. II we briefly describe the theoretical origin of neutrino NSI's in Earth matter. In Sec. III we briefly summarize our analysis of the atmospheric neutrino data in terms of $\nu_{\mu} \rightarrow \nu_{\tau}$ vacuum oscillations. In Sec. IV we update our analysis for the pure NSI mechanism, and we show that the latest data are able to rule it out as the dominant $\nu_{\mu} \rightarrow \nu_{\tau}$ conversion mechanism for atmospheric neutrinos. In Sec. V we therefore investigate the combined situation, where massive neutrinos not only oscillate but may also experience NSI's with matter. In this section we derive limits to the NSI parameters from the atmospheric neutrino data. In Sec. VI we present our conclusions.

\section{THEORY}

Generically models of neutrino mass may lead to both oscillations and neutrino NSI's in matter. Here we sketch two simple possibilities.

\section{A. NSI from neutrino mixing}

The most straightforward case is when neutrino masses follow from the admixture of isosinglet neutral heavy leptons

\footnotetext{
${ }^{3}$ The NSI may, however, be rather small [34].
}

as, for example, in seesaw schemes [36]. These contain $S U(2) \otimes U(1)$ singlets with a gauge invariant Majorana mass term of the type $M_{R i j} \nu_{i}^{c} \nu_{j}^{c}$ which breaks total lepton number symmetry. The masses of the light neutrinos are obtained by diagonalizing the mass matrix

$$
\left[\begin{array}{ll}
M_{L} & D \\
D^{T} & M_{R}
\end{array}\right]
$$

in the basis $\nu, \nu^{c}$, where $D$ is the standard $S U(2) \otimes U(1)$ breaking Dirac mass term, and $M_{R}=M_{R}^{T}$ is the large isosinglet Majorana mass and the $M_{L} \nu \nu$ term is an isotriplet [23]. In $S O(10)$ models the first may arise from a 126 vacuum expectation value, while the latter is generally suppressed by the left-right breaking scale, $M_{L} \propto 1 / M_{R}$.

In such models the structure of the associated weak currents is rather complex [23]. The first point to notice is that the isosinglets, presumably heavy, will mix with the ordinary isodoublet neutrinos in the charged current weak interaction. As a result, the mixing matrix describing the charged leptonic weak interaction is a rectangular matrix $K$ [23] which may be decomposed as

$$
K=\left(K_{L}, K_{H}\right),
$$

where $K_{L}$ and $K_{H}$ are $3 \times 3$ matrices. The corresponding neutral weak interactions are described by a nontrivial matrix [23]

$$
P=K^{\dagger} K
$$

In such models nonstandard interactions of neutrinos with matter are of gauge origin, induced by the non-trivial structures of the weak currents. Note, however, that since the smallness of neutrino mass is due to the seesaw mechanism $M_{\nu \text { eff }}=M_{L}-D M_{R}^{-1} D^{T}$ the condition

$$
M_{L} \ll M_{R},
$$

the magnitude of neutrino NSI's, is expected to be negligible.

However the number $m$ of $S U(2) \otimes U(1)$ singlets is completely arbitrary, so that one may consider the phenomenological consequences of models with Majorana neutrinos based on any value of $m$. In this case one has $3(1+m)$ mixing angles $\theta_{i j}$ and the same number of $C P$ violating phases $\phi_{i j}$ characterizing the neutrino-mixing matrix $K$ $[23,37]$. This number far exceeds the corresponding number of parameters describing the charged current weak interaction of quarks. The reasons are that (i) neutrinos are Majorana particles so that their mass terms are not invariant under rephasings, and (ii) the isodoublet neutrinos mix with the isosinglets. For $m \leqslant 3,3-m$ neutrinos will remain massless, while $2 m$ neutrinos will acquire Majorana masses but may have nonzero NSI's. For example, in a model with $m=1$ one has one light neutrino and one heavy Majorana neutrino in addition to two massless neutrinos [23] whose degeneracy is lifted by radiative corrections.

In contrast, the case $m>3$ may also be interesting because it allows for an elegant way to generate neutrino masses without a superheavy scale, such as in the seesaw case. This 
allows one to enhance the allowed magnitude of neutrino NSI's strengths by avoiding constraints related to neutrino masses. As an example consider the following extension of the lepton sector of the $S U(2) \otimes U(1)$ theory: let us add a set of two two-component isosinglet neutral fermions, denoted by $\nu^{c}{ }_{i}$ and $S_{i}$, in each generation. In this case one can consider the $9 \times 9$ mass matrix [30]

$$
\left[\begin{array}{lll}
0 & D & 0 \\
D^{T} & 0 & M \\
0 & M^{T} & \mu
\end{array}\right]
$$

(in the basis $\nu, \nu^{c}, S$ ). The Majorana masses for the neutrinos are determined from

$$
M_{L}=D M^{-1} \mu M^{T-1} D^{T} .
$$

In the limit $\mu \rightarrow 0$ the exact lepton number symmetry is recovered and will keep neutrinos strictly massless to all orders in perturbation theory, as in the standard model [24]. The propagation of the light (massless when $\mu \rightarrow 0$ ) neutrinos is effectively described by an effective truncated mixing matrix $K_{L}$ which is not unitary. This may lead to oscillation effects in supernovae matter, even if neutrinos were massless $[21,38,39]$. The strength of NSI is therefore unrestricted by the magnitude of neutrino masses, only by universality limits, and may be large, at the few percent level. The phenomenological implications of these models have been widely investigated [40-44].

\section{B. NSI from new scalar interactions}

An alternative and elegant way to induce neutrino NSI's is in the context of unified supersymmetric models as a result of supersymmetric scalar lepton nondiagonal vertices induced by renormalization group evolution $[25,26]$. In the case of $S U(5)$ the NSI may exist without neutrino mass. In $S O(10)$ neutrino masses coexist with neutrino NSI's.

An alternative way to induce neutrino NSI without invoking physics at very large mass scales is in the context of some radiative models of neutrino masses [29]. In such models NSI may arise from scalar interactions.

Here we focus on a more straightforward way to induce NSI based on the most general form of low-energy supersymmetry. In such models no fundamental principle precludes the possibility to violate $R$ parity conservation [27] explicitly by renormalizable (and hence a priori unsuppressed) operators such as the following extra $L$-violating couplings in the superpotential:

$$
\begin{gathered}
\lambda_{i j k} L_{i} L_{j} E_{k}^{c}, \\
\lambda_{i j k}^{\prime} L_{i} Q_{j} D_{k}^{c},
\end{gathered}
$$

where $L, Q, E^{c}$, and $D^{c}$ are (chiral) superfields which contain the usual lepton and quark $S U(2)$ doublets and singlets, respectively, and $i, j, k$ are generation indices. The couplings in Eq. (7) give rise at low energy to the following four-fermion effective Lagrangian for neutrino interactions with $d$ quark's including

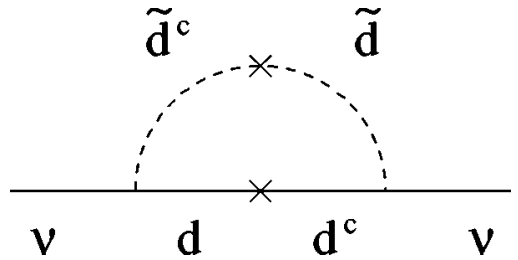

FIG. 1. Diagram generating neutrino mass in supersymmetry with explicitly broken $R$ parity. It illustrates the coexistence of OSC and NSI mechanisms used in Eq. (24).

$$
L_{e f f}=-2 \sqrt{2} G_{F} \sum_{\alpha, \beta} \xi_{\alpha \beta} \bar{\nu}_{L \alpha} \gamma^{\mu} \nu_{L \beta} \bar{d}_{R} \gamma^{\mu} d_{R}, \quad \alpha, \beta=e, \mu, \tau,
$$

where the parameters $\xi_{\alpha \beta}$ represent the strength of the effective interactions normalized to the Fermi constant $G_{F}$. One can identify explicitly, for example, the following nonstandard flavor-conserving NSI couplings:

$$
\begin{gathered}
\xi_{\mu \mu}=\sum_{j} \frac{\left|\lambda_{2 j 1}^{\prime}\right|^{2}}{4 \sqrt{2} G_{F} m_{\tilde{q}_{j L}}^{2}}, \\
\xi_{\tau \tau}=\sum_{j} \frac{\left|\lambda_{3 j 1}^{\prime}\right|^{2}}{4 \sqrt{2} G_{F} m_{\tilde{q}_{j L}}^{2}},
\end{gathered}
$$

and the FC coupling

$$
\xi_{\mu \tau}=\sum_{j} \frac{\lambda_{3 j 1}^{\prime} \lambda_{2 j 1}^{\prime}}{4 \sqrt{2} G_{F} m_{\tilde{q}_{j L}}^{2}},
$$

where $m_{\tilde{q}_{j L}}$ are the masses of the exchanged squarks and $j$ $=1,2,3$ denotes $\widetilde{d}_{L}, \tilde{s}_{L}, \widetilde{b}_{L}$, respectively. Likewise, one can identify the corresponding flavor-changing NSI. The existence of effective neutral current interactions contributing to the neutrino scattering off $d$ quarks in matter provides new flavor-conserving as well as flavor-changing terms for the matter potentials of neutrinos. Such NSI's are directly relevant for atmospheric neutrino propagation. As a final remark we note that such neutrino NSI's are accompanied by nonzero neutrino masses, for example, induced by loops such as that in Fig. 1. The latter lead to vacuum oscillation (OSC) of atmospheric neutrinos. The relative importance of NSI and OSC is model dependent. In what follows we will investigate the relative importance of NSI-induced and neutrino mass oscillation-induced (OSC-induced) conversion of atmospheric neutrinos allowed by the present high statistics data.

\section{VACUUM OSCILLATION HYPOTHESIS}

We first briefly report our updated results for the usual $\nu_{\mu} \rightarrow \nu_{\tau}$ vacuum oscillation channel. For definiteness we confine to the simplest case of two neutrinos, in which case 
TABLE I. Minimum $\chi^{2}$ values and best-fit points for the various atmospheric neutrino data sets considered in the analysis and for two different neutrino conversion mechanisms: pure $\nu_{\mu} \rightarrow \nu_{\tau}$ vacuum oscillation (OSC) and pure nonstandard neutrino-matter interactions (NSI's).

\begin{tabular}{|c|c|c|c|c|c|c|c|c|c|}
\hline \multirow[b]{2}{*}{ Data set } & \multirow[b]{2}{*}{ Degrees of freedom } & \multicolumn{4}{|c|}{$\nu_{\mu} \rightarrow \nu_{\tau}$ oscillations } & \multicolumn{4}{|c|}{ NSI hypothesis } \\
\hline & & $\Delta m^{2}\left[\mathrm{eV}^{2}\right]$ & $\sin ^{2}(2 \theta)$ & $\chi_{\mathrm{OSC}}^{2}$ & GOF & $\varepsilon$ & $\varepsilon^{\prime}$ & $\chi_{\mathrm{FC}}^{2}$ & GOF \\
\hline SK sub-GeV & $10-2$ & $2.2 \times 10^{-3}$ & 1.00 & 4.1 & $84 \%$ & 0.196 & 0.010 & 5.1 & $75 \%$ \\
\hline SK multi-GeV & $10-2$ & $2.1 \times 10^{-3}$ & 0.94 & 4.2 & $84 \%$ & 0.667 & 0.431 & 4.2 & $84 \%$ \\
\hline SK stopping $\mu$ & $5-2$ & $3.0 \times 10^{-3}$ & 0.99 & 0.7 & $88 \%$ & 0.697 & 0.317 & 2.5 & $48 \%$ \\
\hline SK through-going $\mu$ & $10-2$ & $6.3 \times 10^{-3}$ & 0.78 & 5.3 & $73 \%$ & 0.041 & 0.138 & 5.7 & $68 \%$ \\
\hline MACRO & $10-2$ & $1.3 \times 10^{-3}$ & 1.00 & 11.6 & $17 \%$ & 0.020 & 0.046 & 6.6 & $58 \%$ \\
\hline SK contained & $20-2$ & $2.1 \times 10^{-3}$ & 1.00 & 8.8 & $96 \%$ & 0.667 & 0.138 & 10.9 & $90 \%$ \\
\hline SK upgoing & $15-2$ & $3.2 \times 10^{-3}$ & 0.94 & 6.5 & $92 \%$ & 0.041 & 0.144 & 16.5 & $22 \%$ \\
\hline SK contained + stopping $\mu$ & $25-2$ & $2.5 \times 10^{-3}$ & 0.99 & 10.0 & $99 \%$ & 0.697 & 0.331 & 15.3 & $88 \%$ \\
\hline Through-going $\mu$ & $20-2$ & $3.0 \times 10^{-3}$ & 0.95 & 18.1 & $45 \%$ & 0.018 & 0.058 & 21.1 & $28 \%$ \\
\hline SK & $35-2$ & $2.7 \times 10^{-3}$ & 0.97 & 16.2 & $99 \%$ & 0.536 & 0.611 & 53.1 & $1 \%$ \\
\hline $\mathrm{SK}+\mathrm{MACRO}$ & $45-2$ & $2.5 \times 10^{-3}$ & 0.96 & 28.7 & $95 \%$ & 0.513 & 0.667 & 67.6 & $1 \%$ \\
\hline
\end{tabular}

$C P$ is conserved in standard oscillations. ${ }^{4}$ The evolution of neutrinos from the production point in the atmosphere up to the detector is described by the evolution equation

$$
i \frac{d}{d r}\left(\begin{array}{c}
\nu_{\mu} \\
\nu_{\tau}
\end{array}\right)=\mathbf{H}\left(\begin{array}{c}
\nu_{\mu} \\
\nu_{\tau}
\end{array}\right)
$$

where the Hamiltonian which governs the neutrino propagation can be written as

$$
\mathbf{H}=\left(\begin{array}{ll}
H_{\mu \mu} & H_{\mu \tau} \\
H_{\mu \tau} & H_{\tau \tau}
\end{array}\right)=\frac{\Delta m^{2}}{4 E} \mathbf{R}_{\theta}\left(\begin{array}{ll}
-1 & 0 \\
0 & 1
\end{array}\right) \mathbf{R}_{\theta}^{\dagger} .
$$

In Eq. (14) $\Delta m^{2}$ is the squared-mass difference between the two neutrino mass eigenstates and the rotation matrix $\mathbf{R}_{\theta}$ is simply given in terms of the mixing angle $\theta$ by

$$
\mathbf{R}_{\theta}=\left(\begin{array}{ll}
\cos \theta & \sin \theta \\
-\sin \theta & \cos \theta
\end{array}\right) \text {. }
$$

The oscillation probability for a neutrino which travels a path of length $L$ is therefore

$$
P_{\nu_{\mu} \rightarrow \nu_{\tau}}=P_{\nu_{\mu} \rightarrow \bar{\nu}_{\tau}}=\sin ^{2}(2 \theta) \sin ^{2}\left(1.27 \frac{\Delta m^{2} L}{E_{\nu}}\right),
$$

where $\Delta m^{2}, L$, and $E_{\nu}$ are measured in $\mathrm{eV}^{2}, \mathrm{~km}$, and $\mathrm{GeV}$, respectively.

The calculation of the event rates and the statistical analysis is performed according to Ref. [17]. In the present analysis we include the full set of 79 kton yr Super-Kamiokande data [3] and the latest MACRO data on upgoing muons [33]. The results of the fits are shown in Table I: the best-fit point is $\Delta m^{2}=2.7 \times 10^{-3} \mathrm{eV}^{2}$ and $\sin ^{2} 2 \theta=0.97$ with a GOF of

\footnotetext{
${ }^{4}$ In $L$-violating oscillations there is in principle $C P$ violation due to Majorana phases.
}

99\% when only Super-Kamiokande data are considered. The inclusion of MACRO data lowers slightly the GOF to $95 \%$ but practically does not move the best-fit point, which in this case is $\Delta m^{2}=2.5 \times 10^{-3} \mathrm{eV}^{2}$ and $\sin ^{2} 2 \theta=0.96$.

Figure 2 shows the allowed region in the plane $\left(\sin ^{2} 2 \theta, \Delta m^{2}\right.$ ), and Fig. 3 reports the angular distributions of the Super-Kamiokande data sets and the same distributions calculated for the best-fit point. The agreement between the data and the calculated rates in presence of oscillation is remarkable, for each data sample. The same occurs also for the MACRO data set.

From this analysis we can conclude that the $\nu_{\mu} \rightarrow \nu_{\tau}$ oscillation hypothesis represents a remarkably good explanation of the atmospheric neutrino anomaly (see also Refs. $[17,19])$.

\section{NONSTANDARD NEUTRINO INTERACTIONS}

Let us reanalyze the interpretation of the atmospheric neutrino anomaly in terms of pure nonstandard interactions of

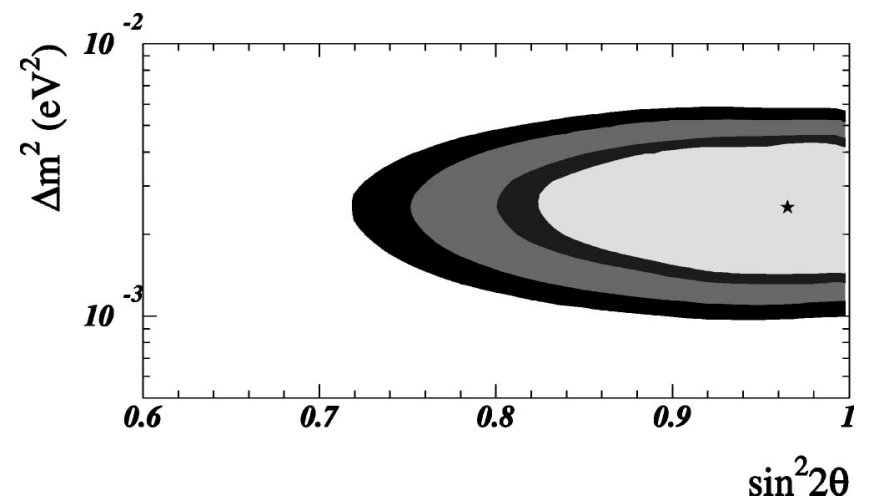

FIG. 2. Allowed regions in the $\Delta m^{2}-\sin ^{2}(2 \theta)$ parameter space for the pure $\nu_{\mu} \rightarrow \nu_{\tau}$ oscillation mechanism. The shaded areas refer to the $90 \%, 95 \%, 99 \%$, and $99.7 \%$ C.L. with two parameters. The best-fit point is indicated by a star. Both Super-Kamiokande and MACRO data have been included. 

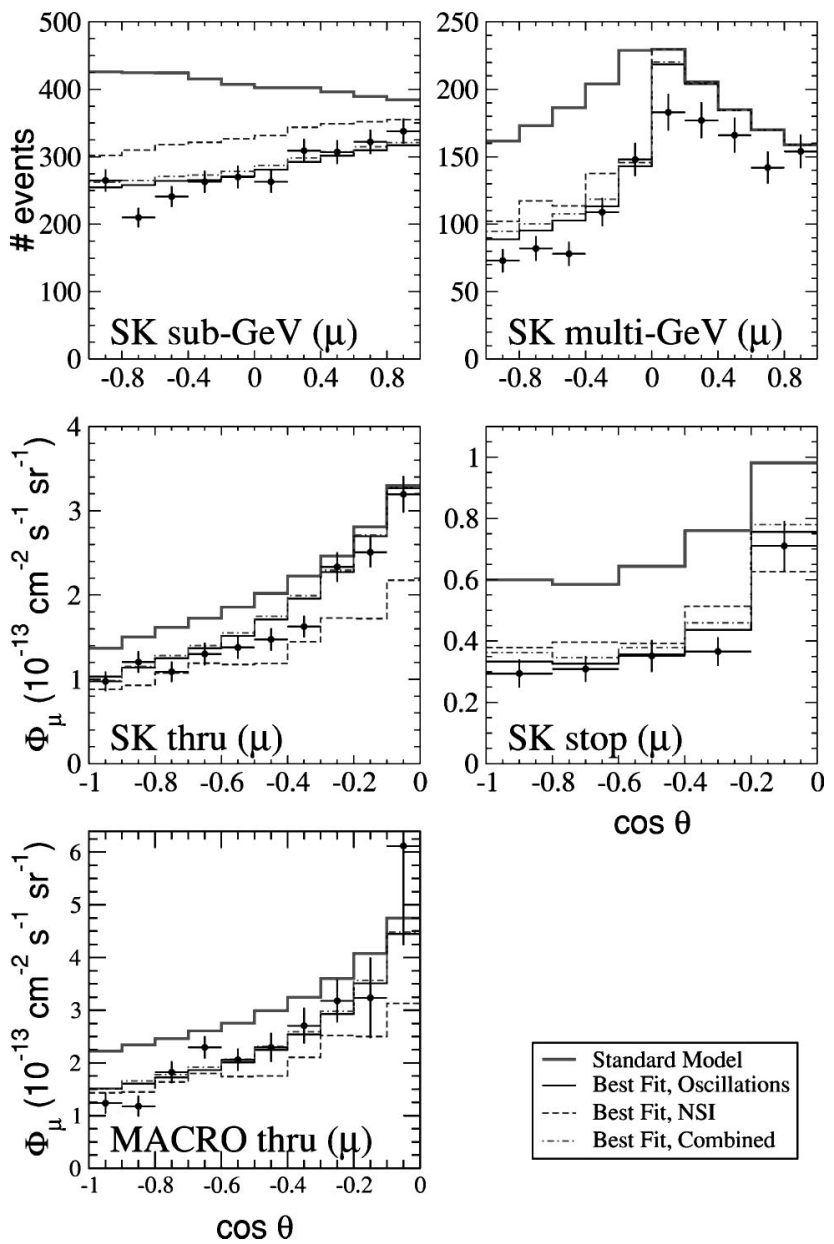

FIG. 3. Zenith-angle distributions for the Super-Kamiokande and MACRO data sets, together with our predictions in the absence of oscillation (thick solid line) and the predictions for the best-fit points for each data set in the different $\nu_{\mu} \rightarrow \nu_{\tau}$ transition channels: pure oscillation (thin solid line), pure NSI (dashed line), and the hybrid oscillation + NSI mechanism (dot-dashed line). The errors displayed in the experimental points are statistical only.

neutrinos with matter $[12,31,32]$. In this case, neutrinos are assumed to be massless and the $\nu_{\mu} \rightarrow \nu_{\tau}$ conversion is due to some NSI with the matter which composes the mantle and the core of the Earth. The evolution Hamiltonian can be written as $[12,31]$

$$
\mathbf{H}= \pm \sqrt{2} G_{F} N_{f}(r)\left(\begin{array}{ll}
0 & \varepsilon_{\nu} \\
\varepsilon_{\nu} & \varepsilon_{\nu}^{\prime}
\end{array}\right),
$$

where the sign $+(-)$ holds for neutrinos (antineutrinos) and $\varepsilon_{\nu}$ and $\varepsilon_{\nu}^{\prime}$ parametrize the deviation from standard neutrino interactions: $\sqrt{2} G_{F} N_{f}(r) \varepsilon_{\nu}$ is the forward scattering amplitude of the $\mathrm{FC}$ process $\nu_{\mu}+f \rightarrow \nu_{\tau}+f$ and $\sqrt{2} G_{F} N_{f}(r) \varepsilon_{\nu}^{\prime}$ represents the difference between the $\nu_{\tau}+f$ and the $\nu_{\mu}+f$ elastic forward scattering amplitudes. The quantity $N_{f}(r)$ is the number density of the fermion $f$ along the path $r$ of the neutrinos propagating in the Earth. To conform to the analyzes of Ref. [12], we set our normalization on these parameters by considering that the relevant neutrino interaction in the Earth occurs only with down-type quarks.

In general, an equation analogous to Eq. (17) holds for antineutrinos, with parameters $\varepsilon_{\nu}^{-}$and $\varepsilon_{\nu}^{\prime}$. For the sake of simplicity, we will assume here and in the following $\varepsilon_{\nu}$ $=\varepsilon_{\nu} \equiv \varepsilon$ and $\varepsilon_{\nu}^{\prime}=\varepsilon_{\nu}^{\prime} \equiv \varepsilon^{\prime}$. It is therefore useful to introduce the following variables $(F, \varphi)$ instead of $\left(\varepsilon, \varepsilon^{\prime}\right)$ :

$$
\begin{aligned}
\varepsilon & =F \sin (2 \varphi), \\
\frac{\varepsilon^{\prime}}{2} & =F \cos (2 \varphi),
\end{aligned}
$$

or, equivalently,

$$
\begin{aligned}
& F=\sqrt{\varepsilon^{\prime 2} / 4+\varepsilon^{2}}, \\
& \varphi=\frac{1}{2} \arctan \left(\frac{\varepsilon}{\varepsilon^{\prime} / 2}\right) .
\end{aligned}
$$

With the use of the variables $F$ and $\theta$, the evolution Hamiltonian Eq. (17) can be cast in a form which is analogous to the standard oscillation one:

$$
\mathbf{H}= \pm \sqrt{2} G_{F} N_{f}(r) F \mathbf{R}_{\varphi}\left(\begin{array}{ll}
-1 & 0 \\
0 & 1
\end{array}\right) \mathbf{R}_{\varphi}^{\dagger}
$$

where $\mathbf{R}_{\varphi}$ assumes the structure of a usual rotation matrix with angle $\varphi$ :

$$
\mathbf{R}_{\varphi}=\left(\begin{array}{ll}
\cos \varphi & \sin \varphi \\
-\sin \varphi & \cos \varphi
\end{array}\right) .
$$

The transition probabilities of $\nu_{\mu} \rightarrow \nu_{\tau}\left(\bar{\nu}_{\mu} \rightarrow \bar{\nu}_{\tau}\right)$ are obtained by integrating Eq. (20) along the neutrino trajectory inside the Earth. For the Earth's density profile we employ the distribution given in Ref. [45] and a realistic chemical composition with proton/nucleon ratio 0.497 in the mantle and 0.468 in the core [46]. Although the integration is performed numerically, the transition probability can be written exactly in a simple analytical form as

$$
P_{\nu_{\mu} \rightarrow \nu_{\tau}}=P_{\nu_{\mu} \rightarrow \bar{\nu}_{\tau}}=\sin ^{2}(2 \varphi) \sin ^{2}(\alpha F L),
$$

where

$$
\alpha=\sqrt{2} G_{F}\left\langle N_{f}\right\rangle
$$

and $\left\langle N_{f}\right\rangle$ is the mean value of $N_{f}(r)$ along the neutrino path. Note that the analytical form in Eq. (22) holds exactly despite the fact that the number density $N_{f}(r)$ varies along the path. The quantity $\alpha$ and the relevant product $\alpha L$ which enters the transition probability in Eq. (22) are plotted in Fig. 4 as a function of the zenith angle $\eta$ and calculated for the Earth's profile quoted above. From Fig. 4 it is clearly visible the sharp change from the mantle to the core densities which occurs for $\cos \eta \sim 0.84$. Notice that the transition probability $P_{\nu_{\mu} \rightarrow \nu_{\tau}}\left(P_{\bar{\nu}_{\mu} \rightarrow \bar{\nu}_{\tau}}\right)$ is formally the same as the expression for vacuum oscillation, Eq. (16), with the angle $\varphi$ playing a role 


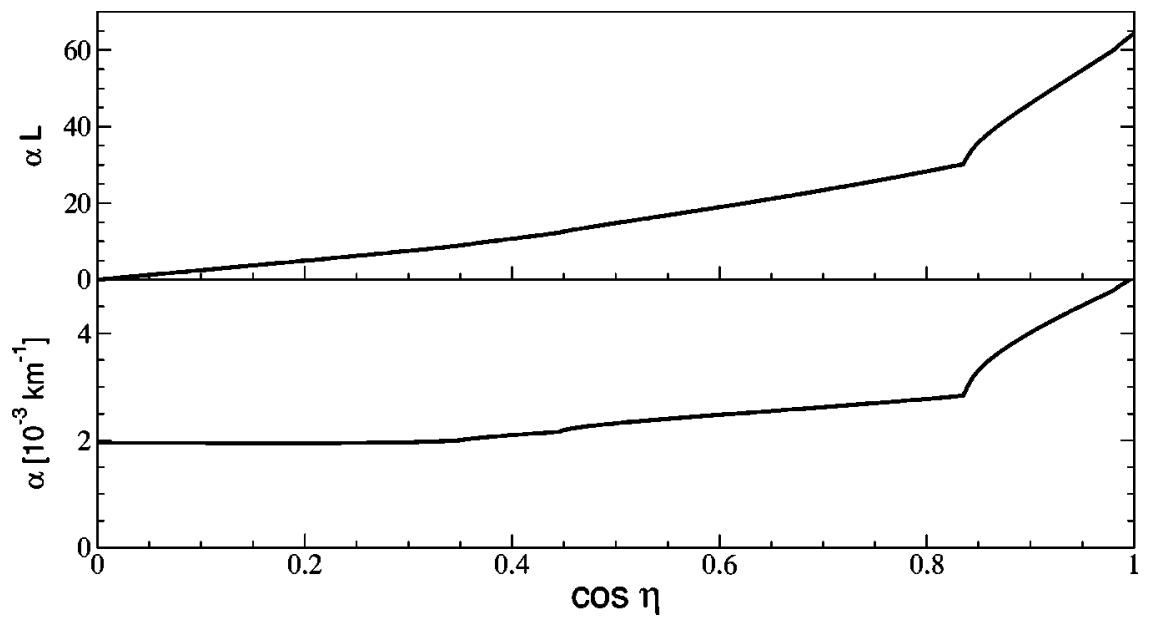

FIG. 4. Function $\alpha$ of Eq. (23) and the relevant product $(\alpha L)$ which enters in the pure NSI transition probability of Eq. (22), plotted as a function of the cosine of the Earth's zenith angle $\eta$.

of mixing angle analogous to the angle $\theta$ for vacuum oscillations. On the other hand, for the factor which depends on the neutrino path $L$, the parameter $F$ formally replaces $\Delta m^{2}$. However, in contrast to the oscillation case, there is no energy dependence in the case of NSI [12,31,32].

The result of the fits to the Super-Kamiokande and MACRO data are reported in Fig. 5 and again in Table I. As already discussed in Ref. [12], the NSI mechanism properly accounts for each Super-Kamiokande (SK) data set separately, as well as the MACRO upgoing muons data. Moreover it succeeds in reconciling together the sub-GeV, multi$\mathrm{GeV}$, and stopping-muons data sets. However, the NSI cannot account at the same time also for the through-going muons events, mainly because the NSI mechanism provides an energy independent conversion probability, while the upgoing muon events, which are originated by higher-energy neutrinos, require a suppression which is smaller than the one required by the other data sets $[12,31,32]$. This effect is clearly visible in two ways. First, from Fig. 5, where we can see that the allowed regions for SK contained + stopping- $\mu$ events (upper-right panel) and for SK + MACRO throughgoing $\mu$ events (lower-left panel) are completely disjoint even at the $99.7 \%$ C.L. In addition, from the angular distribution of the rates shown in Fig. 3, where the angular distribution for upgoing muons calculated for the best-fit point of the pure NSI mechanism clearly shows, too, a strong suppression, especially for horizontal events. The global analysis of Super-Kamiokande and MACRO data has a very low GOF, only 1\%: this now allows us to rule out at $99 \%$ the pure NSI mechanism as a possible explanation of the atmospheric neutrino anomaly.

\section{COMBINING THE OSC AND NSI MECHANISMS}

Let us now consider the possibility that neutrinos are massive and moreover possess nonstandard interactions with matter. As mentioned in Sec. II, this may be regarded as generic in a large class of theoretical models. In this case, their propagation inside the Earth is governed by the following Hamiltonian:

$$
\mathbf{H}=\frac{\Delta m^{2}}{4 E} \mathbf{R}_{\theta}\left(\begin{array}{cc}
-1 & 0 \\
0 & 1
\end{array}\right) \mathbf{R}_{\theta}^{\dagger} \pm \sqrt{2} G_{F} N_{f}(r) F \mathbf{R}_{\varphi}\left(\begin{array}{ll}
-1 & 0 \\
0 & 1
\end{array}\right) \mathbf{R}_{\varphi}^{\dagger}
$$

where $\mathbf{R}_{\theta}$ and $\mathbf{R}_{\varphi}$ are the mixing matrices defined in Eqs. (15) and (21), respectively. The NSI term in the Hamiltonian has an effect which is analogous to the presence of the effective potentials for the propagation in matter of massive neutrinos, a situation which leads to the Mikheyev-SmirnovWolfenstein (MSW) oscillation mechanism [47]. Also in the case of Eq. 24 neutrinos can experience matter-induced oscillations, due to the fact that $\nu_{\mu}$ 's and $\nu_{\tau}$ 's can have both flavor-changing and nonuniversal interaction with the Earth matter.

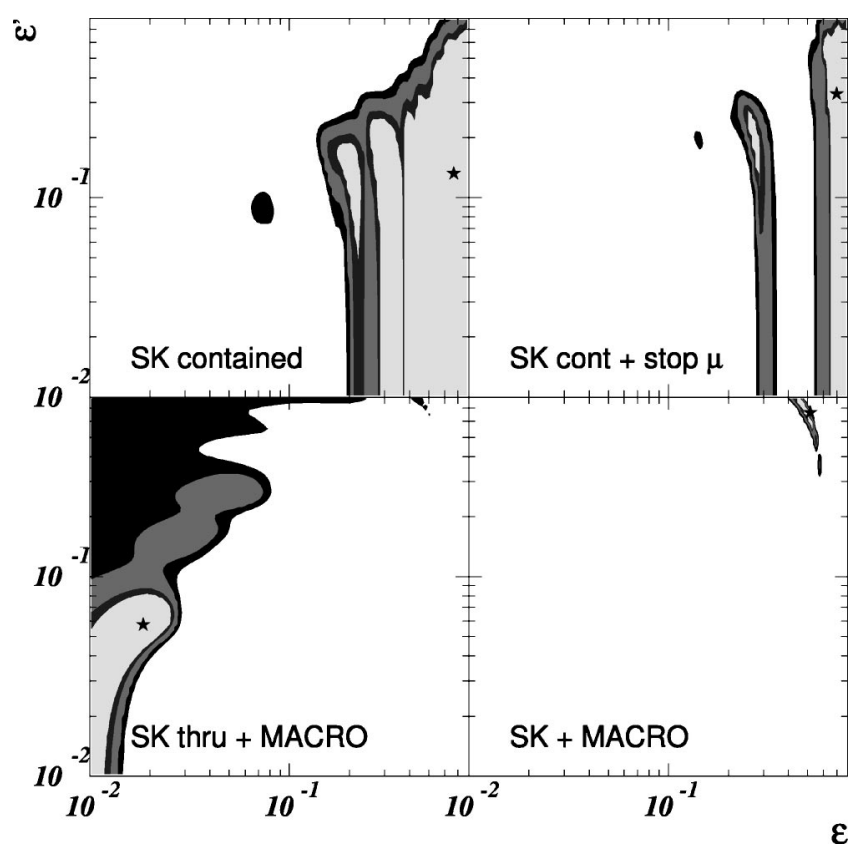

FIG. 5. Allowed regions in the $\varepsilon-\varepsilon^{\prime}$ parameter space for the pure $\nu_{\mu} \rightarrow \nu_{\tau}$ NSI mechanism and for different sets of experimental data. The shaded areas refer to the $90 \%, 95 \%, 99 \%$, and $99.7 \%$ C.L. with two parameters. For each panel, the best-fit point is indicated by a star. 
Since the Earth's matter profile function $N_{f}(r)$ is not constant along the neutrino propagation trajectories, the Hamiltonian matrices calculated at different points inside the Earth do not commute. This leads to a nontrivial evolution for the neutrinos in the Earth, and a numerical integration of Eq. (13) with the Hamiltonian of Eq. (24) is needed in order to calculate the neutrino and antineutrino transition probabilities $P_{\nu_{\mu} \rightarrow \nu_{\tau}}$ and $P_{\bar{\nu}_{\mu} \rightarrow \bar{\nu}_{\tau}}$.

The transition mechanism depends on four independent parameters: the neutrino squared-mass difference $\Delta m^{2}$, the neutrino-mixing angle $\theta$, the FC parameter $\varepsilon$, and the NU parameter $\varepsilon^{\prime}$ (or, alternatively, the $F$ and $\varphi$ parameters for the NSI sector). In our analysis we will use the $F$ and $\varphi$ parameters, which prove to be more useful, and then express the results, which we will obtain for these two parameters, in terms of the $\varepsilon$ and $\varepsilon^{\prime}$ parameters, which have a more physical meaning.

As a first step, we can use the symmetries of the Hamiltonian in order to properly define the intervals of variation of the parameters. Since $\mathbf{H}$ in Eq. (24) is real and symmetric, the transition probabilities are invariant under the following transformations:

$$
\begin{aligned}
\theta & \rightarrow \theta+\pi, \\
\varphi & \rightarrow \varphi+\pi, \\
\Delta m^{2} & \rightarrow-\Delta m^{2} \text { and } \theta \rightarrow \theta+\pi / 2, \\
F & \rightarrow-F \quad \text { and } \quad \varphi \rightarrow \varphi+\pi / 2 .
\end{aligned}
$$

Under any of the above transformations the Hamiltonian remains invariant. Moreover even if the overall sign of the Hamiltonian changes this will have no effect on the calculation of $P_{\nu_{\mu} \rightarrow \nu_{\tau}}$ and $P_{\bar{\nu}_{\mu} \rightarrow \bar{\nu}_{\tau}}$ :

$$
\begin{gathered}
\theta \rightarrow \theta+\pi / 2 \text { and } \varphi \rightarrow \varphi+\pi / 2 \quad(\text { or } \quad \varepsilon \rightarrow \\
\left.-\varepsilon \quad \text { and } \quad \varepsilon^{\prime} \rightarrow-\varepsilon^{\prime}\right) .
\end{gathered}
$$

Finally, if the sign of the nondiagonal entries in the Hamiltonian changes, again there is no effect on the neutrino/ antineutrino conversion probabilities:

$$
\theta \rightarrow-\theta \quad \text { and } \quad \varphi \rightarrow-\varphi \quad(\text { or } \quad \varepsilon \rightarrow-\varepsilon) .
$$

The above set of invariance transformations allows us to define the ranges of variation of the four parameters as follows:

$$
\begin{aligned}
\text { (a) } 0 & \leqslant \theta \leqslant \pi / 4, \\
\text { (b) } 0 & \leqslant \varphi \leqslant \pi, \\
\text { (c) } \Delta m^{2} & \geqslant 0, \\
\text { (d) } \quad F & \geqslant 0 .
\end{aligned}
$$

Notice that, in contrast to the MSW mechanism, it is possible here, without loss of generality, to constrain both the mixing angle $\theta$ inside the $[0, \pi / 4]$ interval keeping $\Delta m^{2}$ positive. There is no "dark side" [48] in the parameter space for this mechanism. ${ }^{5}$ In our analysis we will adopt the set of conditions of Eq. 25, implying that the neutrino squared-mass difference and mixing angle are confined to the same intervals as in the standard $\nu_{\mu} \rightarrow \nu_{\tau}$ oscillation case, while the NSI parameters $\varepsilon$ and $\varepsilon^{\prime}$ can assume independently both positive and negative values. We will actually find that the best-fit point occurs for negative $\varepsilon$ and $\varepsilon^{\prime}$.

Let us turn now to the analysis of the data and the presentation of the results. Here we perform a global fit of the Super-Kamiokande data sets and of the MACRO upgoing muon flux data in terms of the four parameters of the present combined OSC + NSI mechanism. As we have already seen in the previous sections, pure oscillation provides a remarkably good fit to the data, while the pure NSI mechanism is not able to reconcile the anomaly observed in the upgoing muon sample with that seen in the contained event sample. This already indicates that, when combining the two mechanisms of the $\nu_{\mu} \rightarrow \nu_{\tau}$ transition, the oscillation will play the role of leading mechanism, while the NSI could be present at a subdominant level.

As a first result, we quote the best-fit solution: $\Delta m^{2}$ $=2.4 \times 10^{-3} \mathrm{eV}^{2}, \sin ^{2}(2 \theta)=0.99, \varepsilon=-9.1 \times 10^{-3}$, and $\varepsilon^{\prime}$ $=-1.9 \times 10^{-3}$. The goodness of the fit is $94 \%$ (45-4 degrees of freedom). For the $\Delta m^{2}$ and $\sin ^{2}(2 \theta)$ parameters, the best fit is very close to the best-fit solution for pure oscillation (see Table I). This is a first indication that the oscillation mechanism is stable under the perturbation introduced by the additional NSI mechanism. It is interesting to observe that a small amount of FC could be present, at the level of less than a percent, while $\nu_{\mu}$ and $\nu_{\tau}$ interactions are likely to be universal. Moreover, the $\chi^{2}$ function is quite flat in the $\varepsilon$ and $\varepsilon^{\prime}$ directions for $\varepsilon, \varepsilon^{\prime} \rightarrow 0$.

We also display the effect of the NSI mechanism on the determination of the oscillation parameters by showing the result of the analysis in the $\Delta m^{2}$ and $\sin ^{2}(2 \theta)$ plane, for fixed values of the NSI parameters. Figure 6 shows the dependence of the allowed region in the $\Delta m^{2}$ and $\sin ^{2}(2 \theta)$ plane for fixed values of the NSI parameters, in particular for fixed values of $F$ irrespective of the value of $\varphi$, which is "integrated out." Note that for $F \$ 0.02$ the allowed region is almost unaffected by the presence of NSI. For larger values the quality of the fit gets rapidly worse, however the position of the best-fit point in the plane $\left[\sin ^{2}(2 \theta), \Delta m^{2}\right]$ remains extremely stable. For $F \geq 0.1$ the $99 \%$ C.L. allowed region finally disappears. The last panel of Fig. 6 shows the allowed region when both $F$ and $\varphi$ are integrated out. The region obtained is in agreement with the one obtained for the pure oscillation case. We can therefore conclude that the determination of the oscillation parameters $\Delta m^{2}$ and $\sin ^{2}(2 \theta)$ is very stable under the effect of nonstandard neutrino-matter interactions.

We can now look at the results from the point of view of the NSI parameters. This will allow us to set bounds on the maximum allowed level of neutrino NSI. Figure 7 shows the

\footnotetext{
${ }^{5}$ We also notice that one can replace conditions (a) and (b) in Eq. 25 by $\left(\mathrm{a}^{\prime}\right) \quad 0 \leqslant \theta \leqslant \pi$ and $\left(\mathrm{b}^{\prime}\right) \quad 0 \leqslant \varphi \leqslant \pi / 4$. This implies that both $\varepsilon$ and $\varepsilon^{\prime}$ are positive in this case.
} 


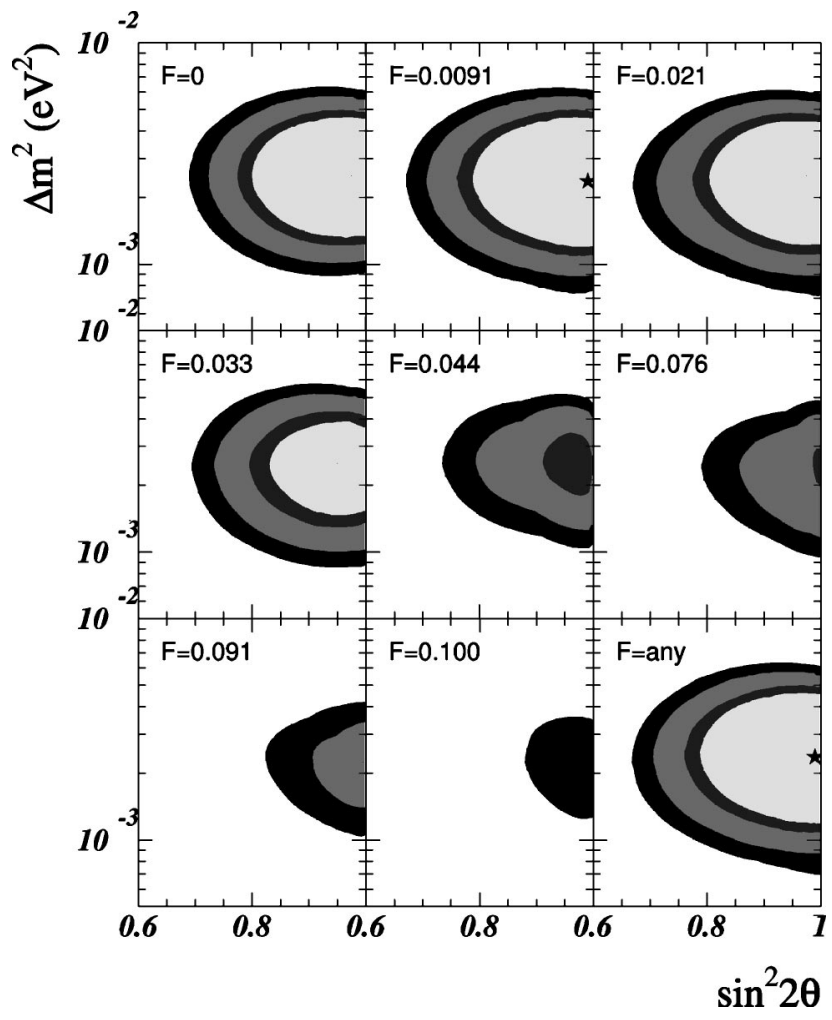

FIG. 6. Allowed regions in the $\Delta m^{2}-\sin ^{2}(2 \theta)$ parameter space for the hybrid OSC + NSI mechanism. In each panel, the value of the NSI parameter $F$ is fixed, while the other NSI parameter $\varphi$ is integrated out. The last panel shows the allowed region when both $F$ and $\varphi$ are integrated out. The shaded areas refer to the $90 \%, 95 \%$, $99 \%$, and $99.7 \%$ C.L. with three parameters, and the best-fit point is indicated by a star. Both Super-Kamiokande and MACRO data have been included.

behavior of the $\chi^{2}$ as a function of the $F$ parameter, and the allowed region in the $F$ and $\varphi$ parameter space with $\Delta m^{2}$ and $\sin ^{2}(2 \theta)$ integrated out. From the lower panel we see that the $F$ parameter is constrained by the data to values smaller than $\sim 0.09$ at $99 \%$ C.L., while the quantity $\varphi$ is not constrained to any specific interval. When $\varphi$ is also integrated out (upper panel of Fig. 7) the number of free parameters is reduced to one, and the upper bound on $F$ improves to $\sim 0.05$.

Looking at Fig. 7 and taking into account the definition of $F$ and $\varphi$ in terms of $\varepsilon$ and $\varepsilon^{\prime}$ given in Eq. (19), we see that the data constrain the maximum amount of FC and NU interactions which is allowed (from $F$ ), but they do not fix their relative amount (through $\varphi$ ). This information can be conveniently translated in the $\varepsilon$ and $\varepsilon^{\prime}$ plane, as we show in Fig. 8: at $99 \%$ C.L., the flavor-changing parameter $\varepsilon$ is confined to $-0.05<\varepsilon<0.04$, while the nonuniversality parameter is bound to $\left|\varepsilon^{\prime}\right|<0.17$. These are the strongest bounds which can be imposed simultaneously on both FC and NU neutrino-matter interactions, but it is also interesting to look at the separate behavior of the $\chi^{2}$ with respect to either FCor NU-type neutrino NSI when the other type of interaction is also integrated out. This is illustrated in Fig. 9, where we

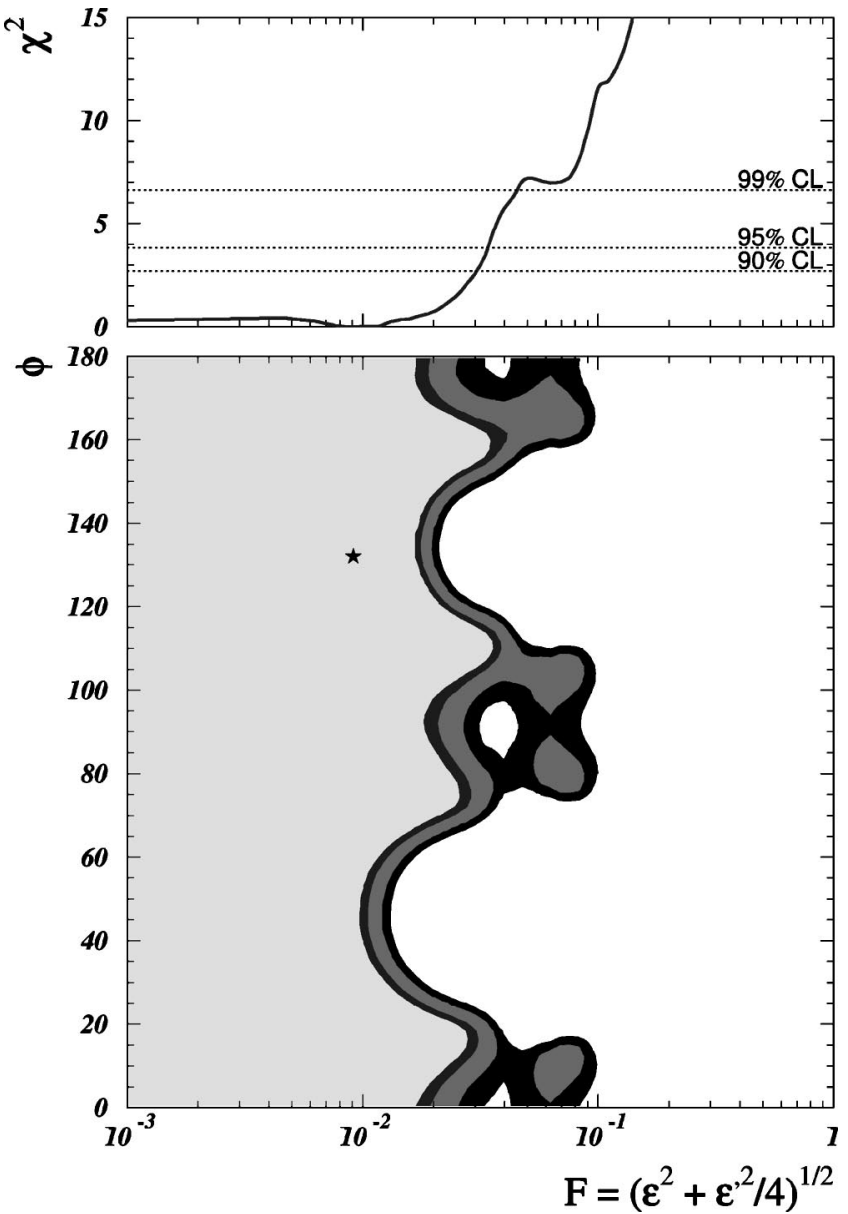

FIG. 7. Allowed regions in the $F-\varphi$ parameter space for the hybrid OSC + NSI mechanism. The parameters $\Delta m^{2}$ and $\sin ^{2}(2 \theta)$ are integrated out. The shaded areas refer to the $90 \%, 95 \%, 99 \%$, and $99.7 \%$ C.L. with two parameters, and the best-fit point is indicated by a star. The top panel shows the behavior of the $\chi^{2}$ as a function of the NSI parameter $F$ when $\varphi$ is also integrated out. Both Super-Kamiokande and MACRO data have been included.

see that the bounds on $\varepsilon$ and $\varepsilon^{\prime}$-now calculated with only one degree of freedom-are improved to $-0.03<\varepsilon<0.02$ and $\left|\varepsilon^{\prime}\right|<0.07$. We also notice that the $\chi^{2}$ function is more shallow for $\varepsilon^{\prime}$ than for $\varepsilon$, indicating that the bound on FC interactions is more stringent than the one on NU interactions.

This is the main result of our analysis, since it provides limits to nonstandard neutrino interactions which are truly model independent, since they are obtained from pure neutrino-physics processes. In particular they do not rely on any relation between neutrinos and charged lepton interactions. Therefore our bounds are totally complementary to what may be derived on the basis of conventional accelerator experiments [49]. Note that although the above bounds of neutrino-matter NSI were obtained simply on the basis of the quality of present atmospheric data, they are almost comparable in sensitivity to the capabilities of a future neutrino factory based on intense neutrino beams from a muon storage ring [50]. 


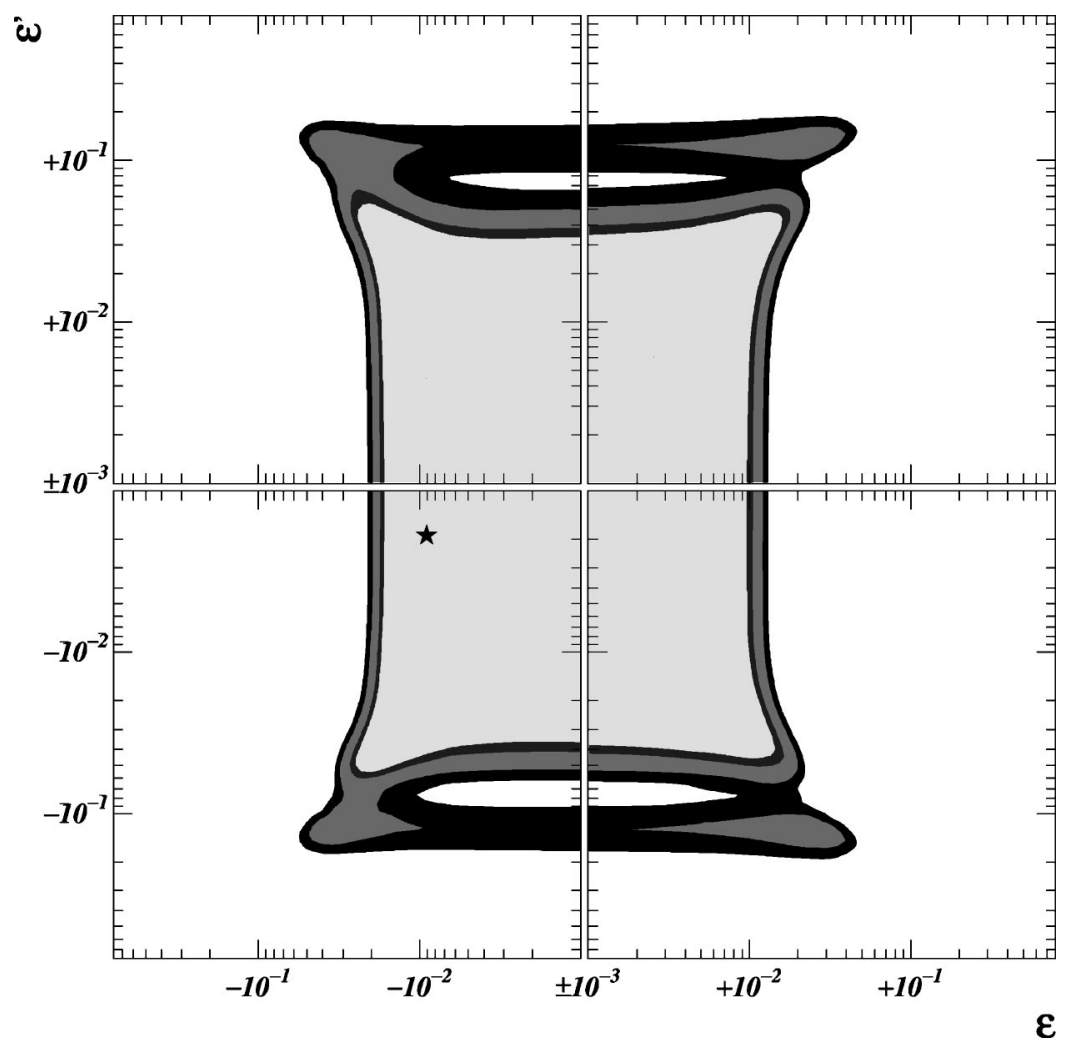

\section{CONCLUSIONS}

In this paper we have analyzed the most recent and large statistic data on atmospheric neutrinos (Super-Kamiokande and MACRO) in terms of three different mechanisms: (i) pure OSC $\nu_{\mu} \rightarrow \nu_{\tau}$ oscillation, (ii) pure NSI $\nu_{\mu} \rightarrow \nu_{\tau}$ transition due to nonstandard neutrino-matter interactions (flavorchanging and nonuniversal), and (iii) hybrid OSC + NSI $\nu_{\mu} \rightarrow \nu_{\tau}$ transition induced by the presence of both oscillation and nonstandard interactions.

The pure oscillation case, as is well known, provides a remarkably good fit to the experimental data, and it can be considered the best and most natural explanation of the atmospheric neutrino anomaly. In this updated analysis, we obtain the best-fit solution for $\Delta m^{2}=2.5 \times 10^{-3} \mathrm{eV}^{2}$ and $\sin ^{2} 2 \theta=0.96$, with a goodness of fit of $95 \%$ (SuperKamiokande and MACRO combined).

In contrast, the pure NSI mechanism, mainly due to its lack of energy dependence on the transition probability, is not able to reproduce the measured rates and angular distributions of the full data sample because it spans about three orders of magnitude in energy. The data clearly show the presence of an up-down asymmetry and some energy dependence. With the increased statistics of the data presently available it is now possible to rule out this mechanism at $99 \%$ as a possible explanation of the atmospheric neutrino data.

We have therefore investigated a more general situation: the possibility that massive neutrinos also possess some amount of flavor-changing interactions with matter, as well as some difference in the interactions between $\nu_{\mu}$ 's and $\nu_{\tau}$ 's. The global analysis of the Super-Kamiokande and MACRO data shows that the oscillation hypothesis is very stable against the possible additional presence of such nonstandard neutrino interactions. The best-fit point is obtained for $\Delta m^{2}$ $=2.4 \times 10^{-3} \mathrm{eV}^{2}, \sin ^{2}(2 \theta)=0.99, \varepsilon=-9.1 \times 10^{-3}$, and $\varepsilon^{\prime}$ $=-1.9 \times 10^{-3}$ with a goodness of fit of $94 \%$ (45-4 degrees
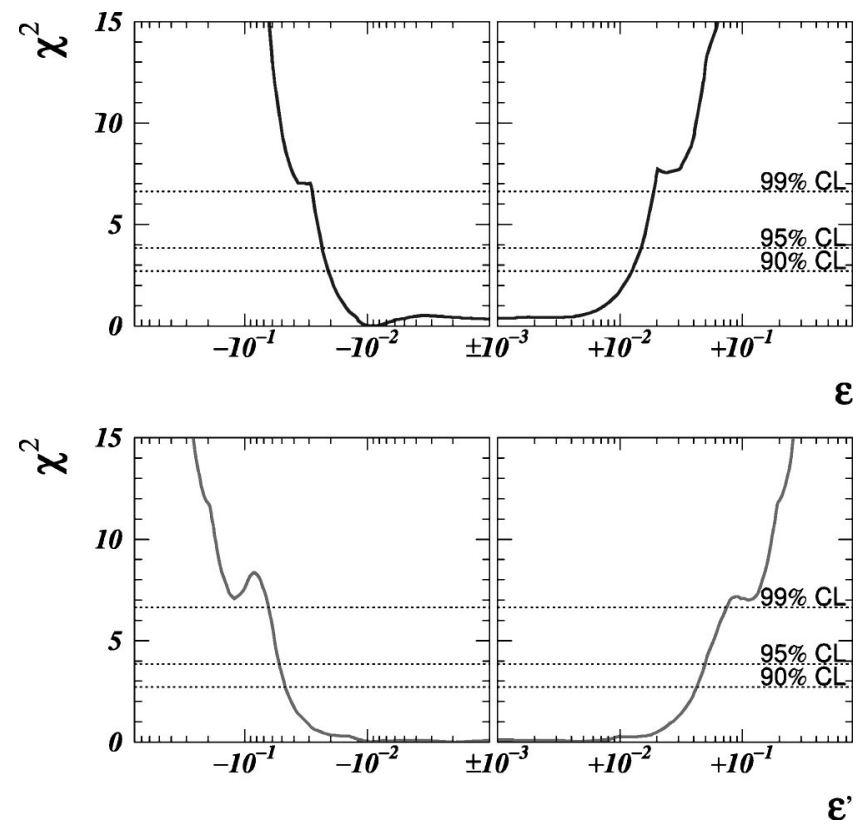

FIG. 9. Behavior of the $\chi^{2}$ as a function of the flavor-changing parameter $\varepsilon$ (top panel) and of the nonuniversal neutrinointeractions parameter $\varepsilon^{\prime}$ (bottom panel), for the hybrid OSC + NSI mechanism. In each panel both the oscillation parameters [ $\sin ^{2}(2 \theta)$ and $\left.\Delta m^{2}\right]$ and the undisplayed NSI parameter $\left(\varepsilon^{\prime}\right.$ in the top panel and $\varepsilon$ in the bottom one) are integrated out. Notice that both positive and negative values of $\varepsilon$ and $\varepsilon^{\prime}$ are shown. 
of freedom). A small amount of FC could therefore be present, at the level of less than a percent, while $\nu_{\mu}$ and $\nu_{\tau}$ interactions are likely to be universal. In addition the $\chi^{2}$ function is rather flat in the $\varepsilon$ and $\varepsilon^{\prime}$ directions for $\varepsilon, \varepsilon^{\prime}$ $\rightarrow 0$ and NSI can be tolerated as long as their effect in atmospheric neutrino propagation is subdominant.

From the analysis we have therefore derived bounds on the amount of flavor changing and nonuniversality allowed in neutrino-matter interactions. At the 99\% C.L., the flavorchanging parameter $\varepsilon$ and the nonuniversality parameter $\varepsilon^{\prime}$ are simultaneously confined to $-0.05<\varepsilon<0.04$ and $\left|\varepsilon^{\prime}\right|$ $<0.17$. The bounds on flavor-changing interactions are stronger than the one which applies to universality violating ones. These bounds on nonstandard neutrino interactions do not rely on any assumption of the underlying particle physics model, as they are obtained from pure neutrino-physics processes. They could be somewhat improved at a future neutrino factory based on intense neutrino beams from a muon storage ring.

Note in particular that the bounds derived here imply that we cannot avoid having a maximal atmospheric neutrinomixing angle $\theta$ by using NSI with nonzero $\varphi$, despite the fact that the value of $\varphi$ is essentially unrestricted. The reason for this lies in the fact that the allowed magnitude of neutrino NSI measured by $F$ is so constrained (due to the lack of energy dependence of the NSI evolution equation) that its contribution must be subleading. This means that a maximum atmospheric neutrino-mixing angle is a solid result which must be incorporated into any acceptable particle physics model, even in the presence of exotic neutrino interactions.

\section{ACKNOWLEDGMENTS}

This work was supported by the Spanish DGICYT under Grant No. PB98-0693, by the European Commission RTN network HPRN-CT-2000-00148, by the European Science Foundation network Grant No. 86, by a CICYT-INFN grant, and by the Research Grants of the Italian Ministero dell'Università e della Ricerca Scientifica e Tecnologica (MURST) within the Astroparticle Physics Project. M.M. is supported by the European Union through Grant No. HPMFCT-2000-01008. N.F. thanks the València Astroparticle and High Energy Physics Group for the kind hospitality. R.T. thanks the Torino Astroparticle Physics Group for hospitality and Generalitat Valenciana for support. We thank also our early collaborators, especially Concha Gonzalez-Garcia, Hiroshi Nunokawa, Todor Stanev, and Orlando Peres, with whom our atmospheric neutrino journey was initiated, see Refs. [12,17,31].
[1] Super-Kamiokande Collaboration, Y. Fukuda et al., Phys. Rev. Lett. 81, 1562 (1998); 82, 2644 (1999).

[2] Kamiokande Collaboration, Y. Fukuda et al., Phys. Lett. B 335, 237 (1994); IMB Collaboration, R. Becker-Szendy et al., Nucl. Phys. B (Proc. Suppl.) 38A, 331 (1995); Soudan Collaboration, W.W.M. Allison et al., Phys. Lett. B 449, 137 (1999); MACRO Collaboration, M. Ambrosio et al., ibid. 434, 451 (1998).

[3] Super-Kamiokande presentations in winter conferences: C. McGrew in Neutrino Telescopes 2001, Venice, Italy, 2001; T. Toshito in Moriond 2001, Les Arcs, France, 2001.

[4] For a review, see T. Kajita and Y. Totsuka, Rev. Mod. Phys. 73, 85 (2001).

[5] B.T. Cleveland et al., Astrophys. J. 496, 505 (1998); Kamiokande Collaboration, Y. Fukuda et al., Phys. Rev. Lett. 77, 1683 (1996); GALLEX Collaboration, W. Hampel et al., Phys. Lett. B 447, 127 (1999); SAGE Collaboration, J.N. Abdurashitov et al., Phys. Rev. Lett. 83, 4686 (1999); SuperKamiokande Collaboration, Y. Fukuda et al., ibid. 81, 1158 (1998).

[6] M.C. Gonzalez-Garcia, M. Maltoni, C. Pena-Garay, and J.W.F. Valle, Phys. Rev. D 63, 033005 (2001); G. L. Fogli, E. Lisi, A. Marrone, D. Montanino, and A. Palazzo, Talk given at Thirty sixth Rencontres de Moriond on Electroweak Interactions and Unified Theories, Les Arcs, France, 2001.

[7] S. Pakvasa, Pramana 54, 65 (2000).

[8] M.C. Gonzalez-Garcia, P.C. de Holanda, C. Pena-Garay, and J.W.F. Valle, Nucl. Phys. B573, 3 (2000).

[9] V. Barger, D. Marfatia, and K. Whisnant, hep-ph/0106207; G.L. Fogli et al., Phys. Rev. D 64, 093007 (2001); J.N. Bah- call et al., J. High Energy Phys. 08, 014 (2001); A. Bandyopadhyay et al., hep-ph/0106264; P. Creminelli et al., J. High Energy Phys. 05, 052 (2001).

[10] O. Miranda et al. Nucl. Phys. B595, 360 (2001); J. Pulido and E. Akhmedov, Phys. Lett. B 485, 178 (2000); M.M. Guzzo and H. Nunokawa, Astropart. Phys. 12, 8 (1999).

[11] S. Bergmann, M.M. Guzzo, P.C. de Holanda, P.I. Krastev, and H. Nunokawa, Phys. Rev. D 62, 073001 (2000).

[12] M.C. Gonzalez-Garcia et al., Phys. Rev. Lett. 82, 3202 (1999).

[13] V. Barger, J.G. Learned, S. Pakvasa, and T.J. Weiler, Phys. Rev. Lett. 82, 2640 (1999); G.L. Fogli, E. Lisi, A. Marrone, and G. Scioscia, Phys. Rev. D 59, 117303 (1999). For particle physics models of unstable neutrinos see Ref. [43].

[14] S. Coleman and S.L. Glashow, Phys. Lett. B 405, 249 (1997); S.L. Glashow, A. Halprin, P.I. Krastev, C.N. Leung, and J. Pantaleone, Phys. Rev. D 56, 2433 (1997).

[15] S. Coleman and S.L. Glashow, Phys. Rev. D 59, 116008 (1999).

[16] M. Gasperini, Phys. Rev. D 38, 2635 (1988); J. Pantaleone, A. Halprin, and C.N. Leung, ibid. 47, 4199 (1993); A. Halprin, C.N. Leung, and J. Pantaleone, ibid. 53, 5365 (1996).

[17] N. Fornengo, M.C. Gonzalez-Garcia, and J.W.F. Valle, Nucl. Phys. B580, 58 (2000); M.C. Gonzalez-Garcia, H. Nunokawa, O.L. Peres, and J.W.F. Valle, ibid. B543, 3 (1999); M.C. Gonzalez-Garcia, H. Nunokawa, O.L. Peres, T. Stanev, and J.W.F. Valle, Phys. Rev. D 58, 033004 (1998).

[18] M. Guzzo et al., Nucl. Phys. B (Proc. Suppl.) 87A, 201 (2000).

[19] R. Foot, R.R. Volkas, and O. Yasuda, Phys. Rev. D 58, 013006 (1998); O. Yasuda, ibid. 58, 091301 (1998); G.L. Fogli, E. 
Lisi, A. Marrone, and G. Scioscia, ibid. 59, 033001 (1999); E.Kh. Akhmedov, A. Dighe, P. Lipari, and A.Yu. Smirnov, Nucl. Phys. B542, 3 (1999).

[20] L. Wolfenstein, Phys. Rev. D 17, 2369 (1978); J. Schechter and J.W.F. Valle, ibid. 22, 2227 (1980).

[21] J.W.F. Valle, Phys. Lett. B 199, 432 (1987).

[22] M. Guzzo, A. Masiero, and S. Petcov, Phys. Lett. B 260, 154 (1991); E. Roulet, Phys. Rev. D 44, 935 (1991); V. Barger, R.J.N. Phillips, and K. Whisnant, ibid. 44, 1629 (1991); S. Bergmann, Nucl. Phys. B515, 363 (1998); E. Ma and P. Roy, Phys. Rev. Lett. 80, 4637 (1998).

[23] J. Schechter and J.W.F. Valle, Phys. Rev. D 22, 2227 (1980).

[24] R. Mohapatra and J.W.F. Valle, Phys. Rev. D 34, 1642 (1986); D. Wyler and L. Wolfenstein, Nucl. Phys. B218, 205 (1983).

[25] L.J. Hall, V.A. Kostelecky, and S. Raby, Nucl. Phys. B267, 415 (1986)

[26] R. Barbieri, L. Hall, and A. Strumia, Nucl. Phys. B445, 219 (1995).

[27] L.J. Hall and M. Suzuki, Nucl. Phys. B231, 419 (1984); G.G. Ross and J.W.F. Valle, Phys. Lett. 151B, 375 (1985); J. Ellis, G. Gelmini, C. Jarlskog, G.G. Ross, and J.W.F. Valle, ibid. 150B, 142 (1985).

[28] S. Bergmann, H.V. Klapdor-Kleingrothaus, and H. Paes, Phys. Rev. D 62, 113002 (2000).

[29] M. Fukugita and T. Yanagida, Phys. Lett. B 206, 93 (1988).

[30] M.C. Gonzalez-Garcia and J.W.F. Valle, Phys. Lett. B 216, 360 (1989).

[31] N. Fornengo, M.C. Gonzalez-Garcia, and J.W.F. Valle, J. High Energy Phys. 07, 006 (2000).

[32] P. Lipari and M. Lusignoli, Phys. Rev. D 60, 013003 (1999); G.L. Fogli, E. Lisi, A. Marrone, and G. Scioscia, ibid. 60, 053006 (1999).

[33] MACRO Collaboration, M. Spurio et al., hep-ex/0101019; MACRO Collaboration, B. Barish, Talk given at Neutrino 2000, 2000, Sudbury, Canada.

[34] M. Hirsch, M.A. Diaz, W. Porod, J.C. Romao, and J.W.F. Valle, Phys. Rev. D 62, 113008 (2000); J.C. Romao et al., ibid.
61, 071703(R) (2000).

[35] S. Bergmann, Y. Grossman, and D.M. Pierce, Phys. Rev. D 61, 053005 (2000).

[36] M. Gell-Mann, P. Ramond, and R. Slansky, in Supergravity, Proceedings of the Workshop, Stony Brook, New York, 1979, edited by P. van Niewenhuizen and D. Freedman (NorthHolland, Amsterdam, 1979); T. Yanagida, in Proceedings of the Workshop on Unified Theories and Baryon Number in the Universe, Tsukuba, Japan, 1979, edited by O. Sawada and A. Sugamoto (KEK Report No. 79-18, Tsukuba, 1979); R.N. Mohapatra and G. Senjanovic, Phys. Rev. Lett. 44, 912 (1980).

[37] J. Schechter and J.W.F. Valle, Phys. Rev. D 23, 1666 (1981).

[38] H. Nunokawa, Y.Z. Qian, A. Rossi, and J.W.F. Valle, Phys. Rev. D 54, 4356 (1996).

[39] D. Grasso, H. Nunokawa, and J.W.F. Valle, Phys. Rev. Lett. 81, 2412 (1998).

[40] J. Bernabeu, A. Santamaria, J. Vidal, A. Mendez, and J.W.F. Valle, Phys. Lett. B 187, 303 (1987).

[41] M.C. Gonzalez-Garcia, A. Santamaria, and J.W.F. Valle, Nucl. Phys. B342, 108 (1990); M. Dittmar, A. Santamaria, M.C. Gonzalez-Garcia, and J.W.F. Valle, ibid. B332, 1 (1990).

[42] N. Rius and J.W.F. Valle, Phys. Lett. B 246, 249 (1990).

[43] J.W.F. Valle, Prog. Part. Nucl. Phys. 26, 91 (1991).

[44] M.C. Gonzalez-Garcia and J.W.F. Valle, Mod. Phys. Lett. A 7, 477 (1992); A. Ilakovac, Phys. Rev. D 62, 036010 (2000).

[45] A.M. Dziewonski and D.L. Anderson, Phys. Earth Planet. Inter. 25, 297 (1981).

[46] J. Bahcall and P. Krastev, Phys. Rev. C 56, 2839 (1997).

[47] L. Wolfenstein, Phys. Rev. D 17, 2369 (1978); S.P. Mikheev and A.Yu. Smirnov, Yad. Fiz. 42, 1441 (1985) [Sov. J. Nucl. Phys. 42, 913 (1985)].

[48] A. de Gouvea, A. Friedland, and H. Murayama, Phys. Lett. B 490, 125 (2000).

[49] Particle Data Group, D.E. Groom et al., Eur. Phys. J. C 15, 1 (2000).

[50] A.M. Gago, M.M. Guzzo, H. Nunokawa, W.J. Teves, and R. Zukanovich Funchal, Phys. Rev. D 64, 073003 (2001). 